\title{
Molecular determinants of chemotherapy resistance in ovarian cancer
}

\author{
"Sequencing analyses in combination with phylogenetic analyses \\ will shed light on the lineage of tumor subclones, allowing novel \\ insights into clonal evolution, cancer progression, disease recurrence \\ and chemotherapy resistance."
}

\begin{abstract}
Keywords: cancer genomics $\bullet$ chemotherapy $\bullet$ functional genomics $\bullet$ heterogeneity - intra-tumor • ovarian cancer • resistance
\end{abstract}

Resistance to chemotherapy is a major contributing factor in treatment failure and consequent mortality associated with cancer [1]. Traditionally, the drivers of chemotherapy resistance were discovered through genetic analysis of cancer cells by comparing the differences in gene expression between chemotherapy sensitive cells and chemotherapy-resistant counterparts made resistant by in vitro exposure to chemotherapeutic agents [1,2]. These studies provided a conceptual framework for wellrecognized mechanisms for chemotherapy resistance: namely pre-target resistance, on-target resistance, post-target resistance and off-target resistance [3].

Pre-target resistance is associated with several mechanisms that affect drug activity and bioavailability before it reaches the target. Pharmacokinetics, hypoxia and stromal composition that affect bioavailability of active drug at the target site could contribute to pre-target resistance. In addition, drug influx transporter, such as Ctr1, and efflux transporters, such as ATP7A and ATP7B, have been implicated in platinum resistance $[4,5]$. Other efflux transporters, such as MDR1 (P-gp) and MRP1, are also implicated in multidrug resistance [2,6-8]. Intracellular glutathione can alter the reactivity of cisplatin and enhance resistance to cisplatin [9]. Finally, recent whole genome tumor sequencing studies indicate that overexpression of MDR1, as a consequence of recurrent promoter fusion in high-grade serous ovarian cancer, is associated with acquired resistance to chemotherapy [10].
Alterations in the molecular target of the drug also contribute to on-target resistance. Cisplatin causes DNA lesions which are in turn recognized by several repair pathways, such as nuclear excision repair, base excision repair and homologous recombination repair. Consequently, defects in DNA repair pathway in cancer cells make them sensitive to cisplatin. However, reversion mutations in BRCA1 and BRCA2 that partially restore homologous recombination repair function are associated with resistance to cisplatin [10-12]. These mutations not only affect response to cisplatin but also sensitize the cells to PARP inhibitors [13]. Similarly, point mutations in the paclitaxel-binding sites on $\alpha$-tubulin and $\beta$-tubulin and selective alterations in $\beta$-tubulin isotypes, such as the overexpression of class III $\beta$-tubulin, have been associated with paclitaxel resistance [14].

Post-target resistance results from multiple alterations within apoptotic pathways, as a result of defects in proapoptotic pathways and/or the aberrant activation of antiapoptotic pathways. One of the best-characterized modes of genotoxic action of cisplatin involves the mitochondrial apoptosis [15]. Consequently, alterations in the factors involved in regulation and execution of apoptosis have the potential to confer chemotherapy resistance [7]. Accumulating evidence have shown that upregulation of antiapoptotic factors such as Bcl-2, Bcl-xL, MCL-1, survivin and downregulation of proapoptotic factors, such as Bax and caspases, correlate with taxane and platinum-based chemotherapy resistance [3,16-19]. FOXO1

\section{Megan Cooley}

Department of Cancer Biology, University of Kansas Medical Center, Kansas City, KS 66160, USA

\section{Pingping Fang}

Department of Cancer Biology, University of Kansas Medical Center, Kansas City, KS 66160, USA

\section{Fang Fang}

Department of Cellular \& Integrative Physiology, Medical Sciences Program, Indiana University, Bloomington, IN 47405, USA

\section{Kenneth P Nephew}

Department of Cellular \& Integrative Physiology, Medical Sciences Program, Indiana University, Bloomington, IN 47405, USA

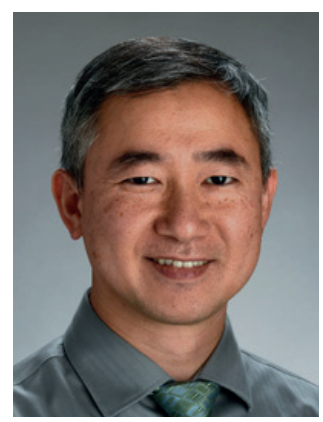

Jeremy Chien

Author for correspondence: Department of Cancer Biology, University of Kansas Medical Center, Kansas City, KS 66160, USA jchien@kumc.edu 
(encoded by FOXO1 gene), a proapoptotic protein was recently shown to be associated with recurrent high-grade serous ovarian cancer (HGSOC) [10].

\begin{abstract}
"In the 'tree' of cancer clonal evolution, chemotherapy may be pruning branches rather than cutting down the trunks, thereby allowing recurrence and resistance to chemotherapy."
\end{abstract}

Off-target resistance is characterized by alterations in the pathways that are not directly engaged by drugs but these alterations nonetheless contribute to resistance. Examples include the overexpression of ERBB2 resulting in elevated prosurvival signaling via PI3K and MAPK, upregulation of heat shock protein and autophagy pathway as reviewed by Galluzzi et al. [3] . In addition, it has been reported that CCNE1 (codes for cyclin E1) amplification is a common event in primary resistant and refractory HGSOC patients $[10,20]$. Also, in the same study, increased expression of genes involved in extracellular matrix structure, cell adhesion and signaling molecular functions were suggested to be associated with short-term relapse in HGSC patients with no CCNE1 amplification [20]. Emerging evidence suggests that tumor microenvironment modulates tumor behavior [21] and the response to chemotherapy [22]. Finally, recent whole-genome sequencing studies indicate inactivation of RB1,NF1 and PTEN in acquired resistance to chemotherapy in HGSOC [10].

\section{Genetics of resistance to targeted therapies}

With the development of new targeted agents, resistant mechanisms to these targeted drugs typically follow the old paradigm. Using functional screens, candidate driver genes for resistance can be uncovered by addressing whether these candidate genes are necessary and sufficient for resistance to targeted therapies, whether these candidate genes are reactivate signaling pathway downstream of the drug targets, and the relevance of candidate genes in clinical resistance. Through these analyses, Garraway and colleagues have shown that pathway reactivation is a common mechanism of resistance to pathway-targeted cancer therapies [23]. For example, c-Raf overexpression can overcome b-Raf inhibition, followed by downstream activation of Mek1/2 [23]. Other growth factor singling pathways that cross-talk with the targeted pathway may also result in the resistance of targeted pathway therapies. For example, downstream transcription factors, upstream G-protein-coupled receptor and PKA are shown to mediate resistance to Ras/Raf pathway inhibitors [24]. Pathway reactivation, pathway bypass, pathway indifference (alternative oncogenic transcriptional activation) are generalized concepts involved in pathway-targeted cancer therapy resistance (summarized in [25]), and these mechanisms can be described as on-target alterations.

\section{Functional genomics}

The open reading frame (ORF) or cDNA library screens are evolving beyond their initial use in the identification of transforming genes. The ORF screens were initially used for genome-scale interrogation of gain-of-function studies to identify genes that may act as oncogenes that drive tumor behavior (see review for detail [26]). These types of screens are being extended to identify drivers of resistance to chemotherapies and targeted therapies. One approach is to use custom, limited ORF library of kinases to identify mechanisms of resistance to a kinase-targeted therapy. This approach led to identification of MAP3K8 as a modulator of Raf inhibitors [23]. Another approach is to use patientderived cDNA libraries transduced into cell lines characterized to be platinum sensitive. Transduced cells are then exposed to cisplatin or paclitaxel, for example, and resistant clones are then selected, expanded, amplified by PCR and sequenced for identification. This approach can also be extended to identify regulators of biological pathways or identification of target pathways with novel drugs (summarized in [27]).

\section{Cancer genomics}

With advances in next-generation sequencing technologies, it is now possible to achieve single-basepair resolution of cancer genomes and observe how these alterations contribute to chemotherapy resistance. Studies reported by Swanton and his colleagues provided genomic insights into clonal heterogeneity within tumor samples and suggested that intratumor heterogeneity may contribute to cancer therapy resistance [28]. In the 'tree' of cancer clonal evolution, chemotherapy may be pruning branches rather than cutting down the trunks, thereby allowing recurrence and resistance to chemotherapy. Genomics analyses provide evidence that patients with heterogeneous tumors consisting of multiple subclonal drivers showed poor overall survival. McGranahan et al. show the presence of subclonal driver mutations that could impact the efficacy of targeted therapies, highlighting the need to target multiple subclonal driver genes to produce durable response to treatment [29]. In addition, there is evidence that chemotherapy, such as Temozolomide, can shape the evolution of cancer subclones that produce recurrence with hypermutated phenotype [30]. Similar studies in ovarian cancer are needed to fully understand how chemotherapy shapes the evolution of cancer subclones and the extent to which clonal heterogeneity contributes to chemotherapy resistance in ovarian cancer. 


\section{Epigenomics}

Epigenetic alterations as potential drivers of chemotherapy resistance is reviewed elsewhere [31]. Prior studies have shown that epigenetic silencing of specific genes, such as HTRA1, SULF1, RASSF1A and $M L H 1$ contribute to chemotherapy resistance [32-35]. By demethylating RASSF1A, MLH1 and ZIC1, we recently demonstrated that the second-generation DNA methylation inhibitor guadecitabine (SGI-110) is a highly effective chemosensitizer in an ovarian cancer mouse xenograft tumor model [36] with clinical potential for patients with recurrent, platinumresistant disease [37]. A recent systematic profiling study of DNA methylation in high-grade serous ovarian cancer reported DNA methylation biomarkers NKD1, VEGFB, PRDX2 significantly associated with response to chemotherapy [38]. In addition, $B R C A 1$ promoter methylation has also been reported to be an important somatic driver in high-grade serous ovar- ian cancers [10,39], and the loss of $B R C A 1$ promoter methylation is associated with acquired resistance to chemotherapy [10]. A strong association of promoter $\mathrm{CpG}$ island hypermethylation and drug-resistant ovarian cancer has been reported [40]. The epigenetic alterations affect the expression of key genes in off-target and post-target mechanisms of chemotherapy resistance, including PI3K/Akt, TGF- $\beta$, cell cycle progression and DNA damage response $[41,42]$.

\section{Perspective}

Multiregion sequencing of primary and recurrent tumor samples would potentially allow better characterization of how chemotherapies shape the evolution of resistance clones (Figure 1). For example, wholeexome sequencing (WXS) and RNA sequencing (RNA-seq) can be performed from multiple spatially discrete tumor or temporally discrete tumor samples. By combining the WXS and RNA-seq, somatic
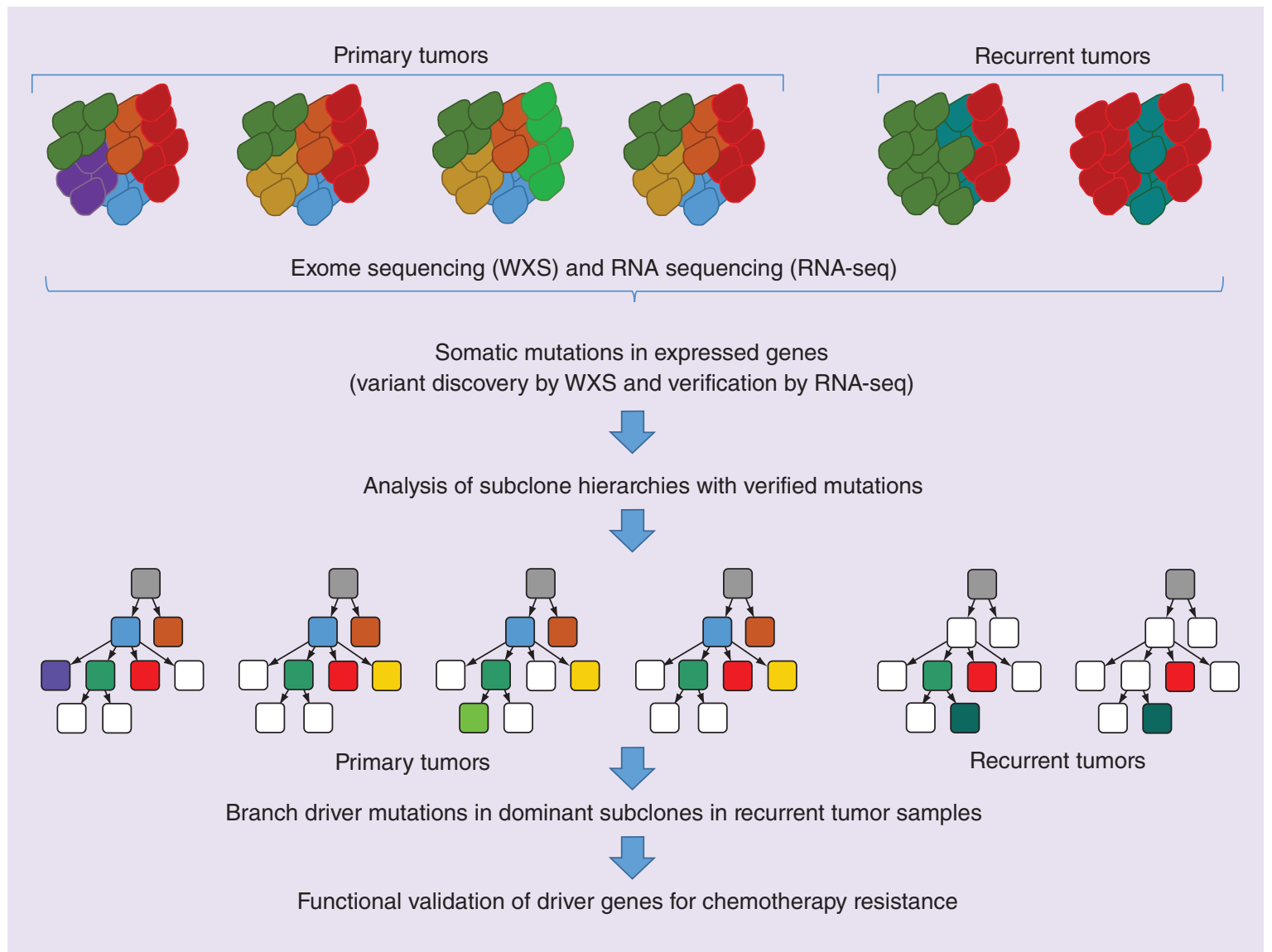

Figure 1. Integrated approach to identify drivers of chemotherapy resistance in ovarian cancer. DNA and RNA sequencing can be used in complementary manner to identify and verify somatic mutations in expressed genes. By applying this approach to spatially and temporally discrete tumor samples from each patient, the constitution, proportional representation and hierarchical structure of cancer subclones at each tumor site can be inferred with phylogenetic analyses. By charactering which subclones are selected by chemotherapy or by identifying which new subclones emerge at the time of recurrence, driver genes associated with these drug-enriched subclones can be identified and functionally validated.

WXS: Whole-exome sequencing. 
mutations in expressed genes can be identified while simultaneously allowing for secondary verification of somatic mutations with RNA-seq. Verified somatic mutations can be used to in phylogenetic clonal inference analyses to reconstruct cancer subclone hierarchies and assess clonal heterogeneity in spatially and temporally discrete tumor samples. These studies will allow the identification of driver mutations in resistant subclones that contribute to chemotherapy resistance.

Clonal heterogeneity is implicated treatment failure and chemotherapy resistance [43]. Sequencing analyses in combination with phylogenetic analyses will shed light on the lineage of tumor subclones, allowing novel insights into clonal evolution, cancer progression, disease recurrence and chemotherapy resistance. In addition and perhaps more importantly, it would be possible to trace the lineage of clonal populations from primary to metastatic tumors and identify which clonal populations are contributing to the clonal heterogeneity and recurrence in each patient. These types of analyses will allow us to identify better therapeutic targets that will be more effective at eliminating heterogeneous

\section{References}

1 Agarwal R, Kaye SB. Ovarian cancer: strategies for overcoming resistance to chemotherapy. Nat. Rev. Cancer 3(7), 502-516 (2003).

2 Gottesman MM. Mechanisms of cancer drug resistance. Annu. Rev. Med. 53, 615-627 (2002).

3 Galluzzi L, Senovilla L, Vitale I et al. Molecular mechanisms of cisplatin resistance. Oncogene 31(15), 1869-1883 (2012).

4 Samimi G, Safaei R, Katano K et al. Increased expression of the copper efflux transporter ATP7A mediates resistance to cisplatin, carboplatin, and oxaliplatin in ovarian cancer cells. Clin. Cancer Res. 10(14), 4661-4669 (2004).

5 Ishida S, Lee J, Thiele DJ, Herskowitz I. Uptake of the anticancer drug cisplatin mediated by the copper transporter Ctr1 in yeast and mammals. Proc. Natl Acad. Sci. USA 99(22), 14298-14302 (2002).

6 Holohan C, Van Schaeybroeck S, Longley DB, Johnston PG. Cancer drug resistance: an evolving paradigm. Nat. Rev. Cancer 13(10), 714-726 (2013).

7 Luqmani YA. Mechanisms of drug resistance in cancer chemotherapy. Med. Princ. Pract. 14(Suppl. 1), 35-48 (2005).

8 Pommier Y, Sordet O, Antony S, Hayward RL, Kohn KW. Apoptosis defects and chemotherapy resistance: molecular interaction maps and networks. Oncogene 23(16), 2934-2949 (2004).

9 Godwin AK, Meister A, O'dwyer PJet al. High resistance to cisplatin in human ovarian cancer cell lines is associated with marked increase of glutathione synthesis. Proc. Natl Acad. Sci. USA 89(7), 3070-3074 (1992).

10 Patch AM, Christie EL, Etemadmoghadam D et al. Wholegenome characterization of chemoresistant ovarian cancer. Nature 521(7553), 489-494 (2015). subclonal populations that contribute to metastasis and chemotherapy resistance. These approaches can be combined with mouse models to identify driver genes in chemotherapy resistance [44]. Finally, as tumor heterogeneity is likely to increase with time, if we want to minimize chemotherapy resistance in the patient population, we must identify cancer at earlier time points when subclonal populations are small and tumor heterogeneity is limited.

\section{Financial \& competing interests disclosure}

This work is supported by the American Cancer Society Research Scholar (125618-RSG-14-067-01-TBE), the Department of Defense Ovarian Cancer Research Program (W81XWH-10-1-0386), and the National Cancer Institute (NCI-R01-CA182832; NCI-U54-CA113011). The authors have no other relevant affiliations or financial involvement with any organization or entity with a financial interest in or financial conflict with the subject matter or materials discussed in the manuscript apart from those disclosed.

No writing assistance was utilized in the production of this manuscript.

11 Edwards SL, Brough R, Lord CJ et al. Resistance to therapy caused by intragenic deletion in BRCA2. Nature 451(7182), 1111-1115 (2008).

12 Sakai W, Swisher EM, Karlan BY et al. Secondary mutations as a mechanism of cisplatin resistance in BRCA2-mutated cancers. Nature 451(7182), 1116-1120 (2008).

13 Lord CJ, Ashworth A. Mechanisms of resistance to therapies targeting BRCA-mutant cancers. Nat. Med. 19(11), 1381-1388 (2013).

14 Mozzetti S, Ferlini C, Concolino P et al. Class III betatubulin overexpression is a prominent mechanism of paclitaxel resistance in ovarian cancer patients. Clin. Cancer Res. 11(1), 298-305 (2005).

15 Cohen SM, Lippard SJ. Cisplatin: from DNA damage to cancer chemotherapy. Prog. Nucleic Acid Res. Mol. Biol. 67, 93-130 (2001).

16 Zaffaroni N, Pennati M, Colella G et al. Expression of the anti-apoptotic gene survivin correlates with taxol resistance in human ovarian cancer. Cell. Mol. Life Sci. 59(8), 1406-1412 (2002).

17 Chaudhry P, Srinivasan R, Patel FD. Expression of the major FAS family and BCL-2 family of proteins in epithelial ovarian cancer (EOC) and their correlation to chemotherapeutic response and outcome. Oncol. Res. 18(11-12), 549-559 (2010).

18 Ziolkowska-Seta I, Madry R, Kraszewska E et al. TP53, BCL-2 and BAX analysis in 199 ovarian cancer patients treated with taxane-platinum regimens. Gynecol. Oncol. 112(1), 179-184 (2009).

19 Williams J, Lucas PC, Griffith KA et al. Expression of Bcl-xL in ovarian carcinoma is associated with chemoresistance and recurrent disease. Gynecol. Oncol. 96(2), 287-295 (2005). 
admoghadam D, Defazio A, Beroukhim R et al. Integrated genome-wide DNA copy number and expression analysis identifies distinct mechanisms of primary chemoresistance in ovarian carcinomas. Clin. Cancer Res. 15(4), 1417-1427 (2009). normal fibroblasts into cancer-associated fibroblasts in ovarian cancer. Cancer Discov. 2(12), 1100-1108 (2012).

Chien J, Kuang R, Landen C, Shridhar V. Platinumsensitive recurrence in ovarian cancer: the role of tumor microenvironment. Front. Oncol. 3, 251 (2013).

23 Johannessen CM, Boehm JS, Kim SY et al. COT drives resistance to RAF inhibition through MAP kinase pathway reactivation. Nature 468(7326), 968-972 (2010). lineage program confers resistance to MAP kinase pathway inhibition. Nature 504(7478), 138-142 (2013).

25 Chien J, Landen CN. Summary of the 2015 American Association for Cancer Research (AACR) Annual Meeting. Gynecol. Oncol. 138(1), 7-10 (2015).

Brummelkamp TR, Bernards R. New tools for functional mammalian cancer genetics. Nat. Rev. Cancer 3(10), 781-789 (2003).

27 Chien J. Functional cancer genomics: a way to bridge the gap between genomic information and biological knowledge. J. Cancer Biol. Res. 1, 1-4 (2013).

28 Burrell RA, Swanton C. Tumour heterogeneity and the evolution of polyclonal drug resistance. Mol. Oncol. 8(6), 1095-1111 (2014).

Mcgranahan N, Favero F, De Bruin EC et al.

Clonal status of actionable driver events and the timing of mutational processes in cancer evolution. Sci. Transl. Med. 7(283), 283 ra254 (2015).

Johnson BE, Mazor T, Hong C et al. Mutational analysis reveals the origin and therapy-driven evolution of recurrent glioma. Science 343(6167), 189-193 (2014).

31 Brown R, Curry E, Magnani L et al. Poised epigenetic states and acquired drug resistance in cancer. Nat. Rev. Cancer 14(11), 747-753 (2014).

Chien J, Staub J, Hu SI et al. A candidate tumor suppressor HtrA1 is downregulated in ovarian cancer. Oncogene 23(8), 1636-1644 (2004).
33 Chien J, Aletti G, Baldi A et al. Serine protease HtrA1 modulates chemotherapy-induced cytotoxicity. J. Clin. Invest. 116(7), 1994-2004 (2006).

34 Staub J, Chien J, Pan Y et al. Epigenetic silencing of HSulf-1 in ovarian cancer: implications in chemoresistance. Oncogene 26(34), 4969-4978 (2007).

35 Matei D, Fang F, Shen C et al. Epigenetic resensitization to platinum in ovarian cancer. Cancer Res. 72(9), 2197-2205 (2012).

36 Fang F, Munck J, Tang J et al. The novel, small-molecule DNA methylation inhibitor SGI-110 as an ovarian cancer chemosensitizer. Clin. Cancer Res. 20(24), 6504-6516 (2014).

37 Fang F, Cardenas H, Miller D et al. Epigenome and genome alterations in platinum resistant ovarian tumors. J. Clin. Oncol. 33 (suppl.; abstract 5555) (2015).

38 Dai W, Zeller C, Masrour N, Siddiqui N, Paul J, Brown R. Promoter $\mathrm{CpG}$ island methylation of genes in key cancer pathways associates with clinical outcome in high-grade serous ovarian cancer. Clin. Cancer Res. 19(20), 5788-5797 (2013).

39 Geisler JP, Hatterman-Zogg MA, Rathe JA, Buller RE. Frequency of BRCA1 dysfunction in ovarian cancer. J. Natl Cancer Inst. 94(1), 61-67 (2002).

40 Wei SH, Balch C, Paik HH et al. Prognostic DNA methylation biomarkers in ovarian cancer. Clin. Cancer Res. 12(9), 2788-2794 (2006).

41 Li M, Balch C, Montgomery JS et al. Integrated analysis of DNA methylation and gene expression reveals specific signaling pathways associated with platinum resistance in ovarian cancer. BMC Med. Genomics 2, 34 (2009).

42 Teodoridis JM, Hall J, Marsh S et al. CpG island methylation of DNA damage response genes in advanced ovarian cancer. Cancer Res. 65(19), 8961-8967 (2005).

43 Gerlinger M, Swanton C. How Darwinian models inform therapeutic failure initiated by clonal heterogeneity in cancer medicine. Br. J. Cancer 103(8), 1139-1143 (2010).

44 Clohessy JG, Pandolfi PP. Mouse hospital and co-clinical trial project-from bench to bedside. Nat. Rev. Clin. Oncol. 12(8), 491-498 (2015). 